\title{
Study on a Spectral Energy Led Lighting of A New Green Plant Breeding System
}

\author{
Zheng liang ${ }^{1,2}, \mathrm{Xu} \mathrm{Wei}^{1,{ }^{*}}, \mathrm{Jiannan} \mathrm{Yuan}^{3}$ and Yanpeng $\mathrm{Li}^{1}$ \\ ${ }^{1}$ JiLin Agricultural Science and Technology University, Jilin, China,132101 \\ ${ }^{2}$ Changchun University of science and Technology,Changchun, Jilin , China,130022 \\ ${ }^{*}$ Changchun Vocational institute technology,Changchun , Jilin, China, 130033
}

Keywords: Led breeding; Spectral energy; Modern agricultural; Multi LED capacity

\begin{abstract}
Semiconductor light emitting diode (LED)have many advantages, such as highly efficient, environmentally friendly, suitable lighting strategies and intelligent light control methods. It solves the constraints of agricultural production activities through flexible light environment configuration in plant growth, poultry breeding and aquatic life. Another Application, LED can kill pest and defense disease, water environmental protection to achieve the purpose of increasing production, high efficiency, high quality, disease resistance and the most important is no pollution. This will increase agricultural product output capacity, improve people's livelihood, safeguard agricultural products, promote social harmony and stability, and accelerate the development of modern agriculture in China. It has very important practical significance. This paper main research a detailed analysis of the structure of the strawberry fill light, and a detailed analysis of the spectrum of the different distances between the fill light and the plant. At last, we will give the final test results.
\end{abstract}

\section{Introduction}

In foreign countries, in 2010, Bula et al. used a 660nm red LED and a blue fluorescent lamp to conduct experiments on the cultivation of plant germs and achieved success. In 2011, the research and development center of the university of Karnataka used red light and blue light to study the differentiation of young shoots of the genus Lilium. The results showed that red-blue LED light irradiation can promote the differentiation of tiger teeth and more suitable for seedling growth [1]-[2]. In China, the Infrared Research Institute of Harbin Engineering University has been devoted to the study of high-efficiency and energy-saving supplements in greenhouses, and has developed High-efficient energy-saving greenhouse rare-earth lamps to solve the key problems in the cultivation of counter-season crops in greenhouses. The effect of the changes on the germination and growth of the plant seed requires further research [3]. For example, application number: 201510769588.1 discloses an LED plant growth lamp with continuous spectrum, including an LED light source lamp bead group, a light source substrate, a radiator, and a light source matching Drive power, LED light source lamp bead group consists of warm white LED and red LED. The correlated color temperature of the warm white LED is below $4000 \mathrm{~K}$. The blue light chip with a light emitting wavelength of 420-490 nanometers (nm) is covered with a phosphor, wherein the phosphor is a YAG phosphor with a light emitting peak of yellow and the peak wavelength is green. The phosphor is made by mixing light, and the ratio of the green phosphor to the total weight of the phosphor is $5 \%$ to $30 \%$. The red LED wavelength is between 620 and 680 nanometers (nm) [4]-[6]. The technical effect of the present invention is to provide effective light promotes plant growth.

An LED plant growth lamp having a wavelength between 430 and 680 nanometers $(\mathrm{nm})$ and having a 
continuous spectrum can satisfy the plant's demand for light of other wavelengths, the light-filling effect is better, and the quality of the plant growth is higher. Energy saving and low production costs [7], although this type of LED lamp can emit light of all spectra, it is impossible to study which spectrum is more effective.

The complexity of agricultural lighting determines the difficulty of experimentation in agricultural lighting research. It is reflected in the specific experiments that there are too many variables in the experiment. Although the main test light conditions, through the study of different light conditions, such as irradiation intensity, spectrum, irradiation time, irradiation cycle, irradiation methods and other conditions in the case of animal and plant growth, It design to fix other agricultural conditions such as temperature, humidity, air, Water, nutrients, seeds, etc. However, these two types of factors may influence each other. Therefore, in the actual experimental study, there are many conditions for experimental changes to be considered. At the same time, each condition has a certain sample for the consideration of biostatistics [8]-[11]. It results in many test conditions and many samples. In addition, each test condition requires a certain growth time. Finally, the situation of agricultural lighting research is: there are many test samples and the test time span. Agricultural lighting has become time-consuming and laborious. For example, if you set three kinds of lighting condition variables, such as spectrum, intensity, irradiation time, each variable uses ten discrete values, then you know that there are one thousand conditions, plus ten biological conditions if it is one billion kinds In case, if there are ten samples in each case, that is one billion samples. If each sample is to be carried out for two months, you can imagine the difficulty of this experiment [12]. Therefore, the actual research was forced to reduce the number of test variables and reduce the number of each test variable.

The advantage of this design is that: this kind of light-filling device can change the spectral parameters of the LED lamp, set the corresponding control set for the spectral setting experiment, and the temperature-humidity sensor monitors the temperature and humidity through the heating rod, humidifier and refrigeration device [13]. The temperature and humidity are controlled in a range suitable for the growth of the seed, and the timer can also record the time of germination during the experiment [14]-[16].

\section{Structure design of plant breeding system}

Mechanism of the breeding system. Among them: 1 - control panel, 2 - support foot, 3 - culture warehouse, 4 - partition, 5 - support board, 6 - timer, 7 - touch screen display, 8 - microprocessor, 9 temperature and humidity sensor ,10 - Refrigerating unit, 11 - Dimming driver, 12 - Infrared emission lamp, 13 - Spectrum-adjustable LED red light, 14 - Spectrum-adjustable LED blue light, 15 - Spectrum test apparatus, 16 - Optical parameter test apparatus, 17 - Illumination test device, 18 - humidifier, 19 heating rod, 20 - LED light group, 21 - glass door.

Fig. 1 is a front view of the present invention.

Fig. 2 is a top view of a control panel in the present invention.

Fig. 3 is a schematic view of an LED lamp assembly in the present invention. 


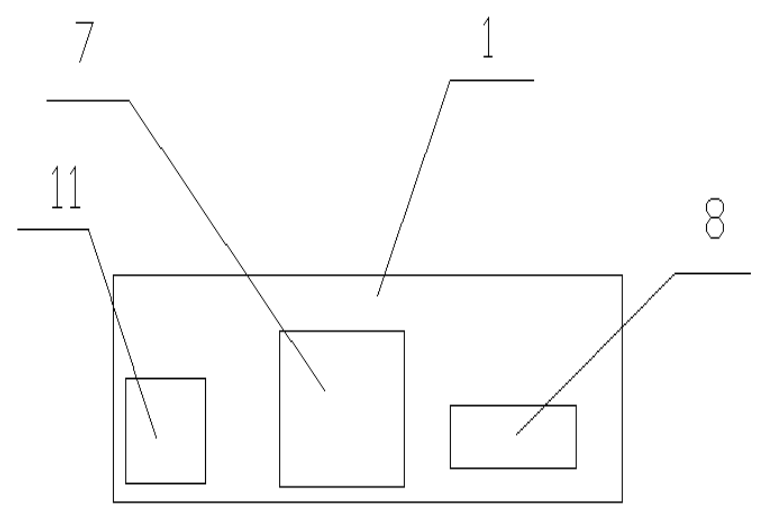

Fig. 1 Structure Diagram 1

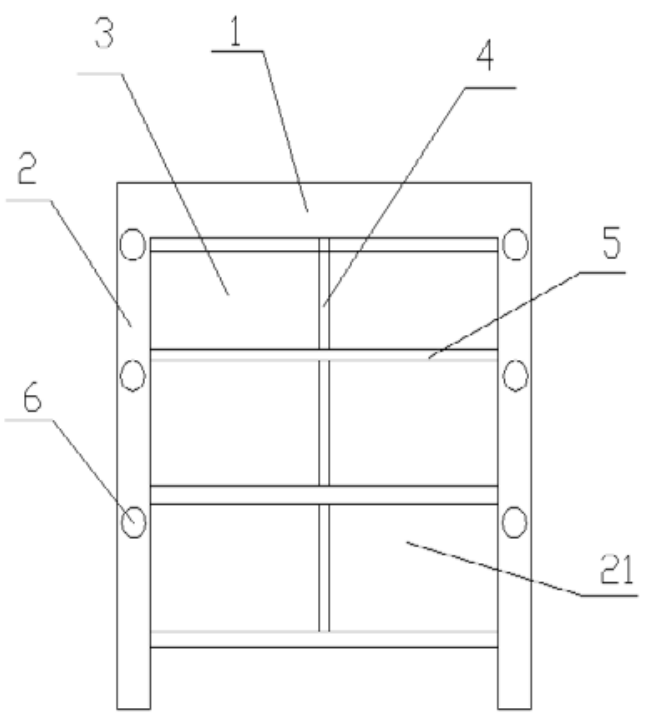

Fig. 2 Structure Diagram 2

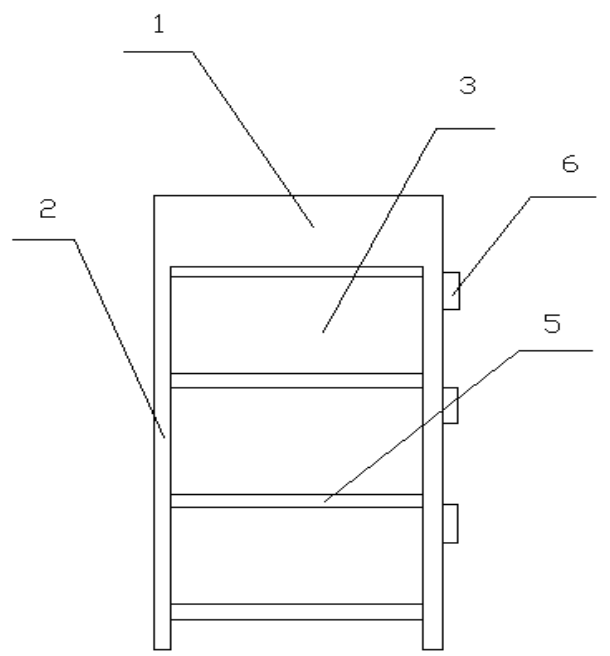

Fig. 3 Structure Diagram 3

As shown in Fig. 1 to Fig. 3 , a spectral energy LED light-up device of a novel green plant includes a light-filling device body. The light-supplying device includes a control panel 1 and a supporting foot 2, 
and a control panel 1 is provided with a dimming driver 11 . The touch display screen 7 and the microprocessor 8 , the dimming driver 11 is used to adjust the spectrum of the LED lamp, and the touch display screen 7 is used to display the change of the temperature and humidity in the culture bin 3 and the linear change of the spectrum, and supports the gap of the foot 2 a plurality of layers of supporting plates 5 are arranged between the supporting plate 5 and a partition plate 4 is vertically arranged in the middle of the supporting plate 5 , and a bunker 3 is formed between the partition plate 4 and the supporting plate 5, and a plurality of culture shelves 3 are provided on the top surface thereof. The LED lamp group 20, at the bottom of the cultivating chamber 3, is provided with a spectrum testing device 15, an optical parameter testing device 16, an illuminance testing device 17, and a heating rod 19. Before the experiment, the spectrum testing device 15, the optical parameter testing device 16, and the illuminance testing device 17 are passed. The light is primary tested to determine the experimental environment. The inside of the supporting plate 5 includes a temperature and humidity sensor 9 , a humidifier 18, and we designed a refrigerating device 10 for maintaining the environment inside the culture chamber 3 , preventing other environmental factors from affecting the experimental results, and dimming. The driver 11 is connected with the LED lamp group 20 The touch display screen 7 is connected with the spectrum test device 15, the optical parameter test device 16, and the illumination test device 17. The test results are displayed to facilitate confirmation of the environmental parameters. The microprocessor 8 and the touch display 7, the heating rod 19, and the temperature and humidity sensor 9. The humidifier 18 and the refrigerating device 10 facilitate the microprocessor 8 to monitor and control the environment within the cultivating chamber 3.

\section{Spectral analysis}

Optical spectrum analysis. The spectrum of LED plant lights is the focus on discussion because the spectrum of the LED light source can be designed according to the planting process requirements. At the same time, the spectrum of the LED light source can achieve variable spectrum control through the dimming technology. The LED light source is currently the only one that can be realized. The variable-spectrum plant light source and plant lamp variable-spectrum technology are mainly aimed at light form control, and have little effect on energy conservation. Ordinary plant lights can also save energy through adjustment of the photo period, and the application cost of variable spectrum will increasing.

\section{Experimental result}

Effect of spectral range on plant physiology. such as during 280 315nm: minimal effect on morphology and physiological processes,during315 400nm: Absorption of chlorophyll, affecting photo periodic effects, preventing stem elongation,during400 520nm (blue): The absorption ratio of chlorophyll and carotenoids is the largest and has the greatest effect on cultivate photosynthesis,during520 610nm(green): Absorption rate of pigment is not high,during610 720nm (red): Absorption rate of chlorophyll is low and has a significant effect on photosynthesis and photo periodic effects,during720 1000nm: Low Absorption Rate, Stimulates Cell Prolongation, Affects Flowering and Seed Germination, If greater than 1000nm : Convert to Heat.

\section{Conclusion and future work}

In this paper, From the above data, the different wavelengths of light on the photosynthesis of plants are different, the light required for plant photosynthesis, the wavelength of 400 720nm. Light from 400 to $520 \mathrm{~nm}$ (blue) and 610to720nm (red) contribute the most to photosynthesis. Light from 520 to $610 \mathrm{~nm}$ (green) is absorbed by plant pigments at a very low rate. 
In accordance with the above principles, plant lights are basically made in three forms of red-blue combination, all-blue, and full-red, to provide two wavelengths of red and blue light, covering the wavelength range required for photosynthesis. In terms of visual effects, the red and blue combination plant lights appear pink.

\section{Acknowledgement}

This research is supported by Youth Project Foundation of JiLin Agricultural Science and Technology University (Ji Nong He Zi [2017]No.111).

\section{References}

[1] Alston, J., Chan-Kang, C., Marra, M., Pardey, P., \& Wyatt, T. (2000). A meta-analysis of rates of return to agricultural R\&D: Ex Pede Herculem? IFPRI Research Report 113. IFPR, Washington, DC Avilla and Evenson, 1995.

[2] Chen, S., \& Ravallion, M. (2000). How did the poorest fare in the 1990's? World Bank, Washington, DC Datt and Ravallion, 1996.

[3] G. Datt, M. Ravallion Farm productivity and rural poverty in India Journal of Development Studies, 34 (4) (1998), pp. 62-85.

[4] A. de Janvry, E. Sadoulet Rural poverty in Latin America: determinants and exit paths Food Policy, 25 (2000), pp. 389-409

[5] Anmer, L., \& Nashchold, F. (2000). Attaining the international development targets: will growth be enough? DFID working paper. DFID, London Hazell and Haddad, 2001.

[6] Hazell, P., \& Haddad, L. (2001). Agricultural research and poverty reduction, food, agriculture and the environment, Discussion paper 34, IFPRI/Technical Advisory Committee of the CGIAR, Washington, DC IFAD, 2001. 